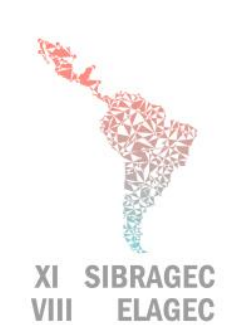

VIII ELAGEC

\section{SIMPÓSIO BRASILEIRO DE GESTÃO E ECONOMIA DA CONSTRUÇÃO}

VIII ENCUENTRO LATINOAMERICANO DE GESTIÓN

Y ECONOMÍA DE LA CONSTRUCCIÓN

Do conhecimento à ação: práticas avançadas de gestão da produção

Londrina, Paraná, Brasil. 23 a 25 de Outubro de 2019

\title{
CONTRIBUIÇÃO DA EXTENSÃO PARA A CONSTRUÇÃO DO GUIA PMBOK® PARA O PLANEJAMENTO DE MÉDIO PRAZO
}

\author{
ANGELIM, Vanessa Lira (1); BARROS NETO, José de Paula (2)
}

(1) Universidade Federal do Ceará, (85) 981524067, e-mail: angelim.vanessa@gmail.com (2) Universidade Federal do Ceará, e-mail: barrosneto@gercon.ufc.br

\begin{abstract}
The Project Management Body of Knowledge (PMBOK®) guide brings together best practices for project management and edited an extension called Construction Extension to the PMBOK® Guide. The medium-term planning level (MTL) is one of the hierarchical levels of the Last Planner System (LPS®) and involves the process of constraint analysis. The present work sought to analyze the contributions of the extension for the construction of the PMBOK® guide for the MTL with the objective of identifying important practices that support the realization of the MTL in the construction. The interactions and contributions of the twelve areas of knowledge of the extension of the PMBOK® guide in the construction with the MTL were pointed out. It was noted that the scope of the MTL permeates and interacts with the management of the twelve areas of knowledge defined by the extension of the PMBOK® Guide in construction. The MTL incorporates actions present in some areas of knowledge or receives input data from others to feed the MTL. The contributions of this paper were to add other categories of constraints to be managed in PMP and to highlight integrated change control and risk management in PMP.
\end{abstract}

Keywords: Medium term planning. Last Planner System. Construction Extension to the PMBOK® Guide.

\section{INTRODUÇÃO}

O guia Project Management Body of Knowledge (PMBOK®) reúne as melhores práticas para o gerenciamento de projetos organizado pelo Project Management Institute (PMI). Para abordar aspectos particulares da indústria da construção, o PMI editou extensão denominada Construction Extension to the PMBOK® Guide (PMI, 2016).

O Last Planner System ${ }^{\circledR}$ (LPS $\left.{ }^{\circledR}\right)$ é um modelo de planejamento na construção, que se baseia em princípios básicos da construção enxuta, desenvolvida por Ballard (2000) e consiste na hierarquização do planejamento em horizontes de longo, médio e curto prazo (BALLARD, 2000). No Planejamento de Médio Prazo (PMP) são identificadas as tarefas que devem ser executadas, os respectivos prazos e são analisadas as restrições das tarefas. (BALLARD; TOMMELEIN, 2016).

Nesse sentindo, o presente trabalho buscou analisar as contribuições da extensão para a construção do guia PMBOK ${ }^{\circledR}$ para o processo de PMP do LPS ${ }^{\circledR}$ com objetivo de identificar práticas importantes que apoiem à realização do PMP na construção. 


\section{SIBRAGEC - ELAGEC 2019 - de 23 a 25 de Outubro - LONDRINA - PR}

\section{REVISÃO BIBLIOGRÁFICA}

O guia PMBOK® é composto por orientações para a gestão de dez áreas de conhecimentos: integração, escopo, cronograma, custo, qualidade, recursos, comunicações, riscos, aquisições e partes interessadas (PMI, 2017). Do ponto de vista estrutural, foram incluídas na extensão para a construção do guia PMBOK® duas áreas de conhecimento adicionais inerentes à construção: Gerenciamento da Saúde, Segurança, Proteção e Meio Ambiente do projeto, e Gerenciamento Financeiro do Projeto (PMI, 2016).

A $6{ }^{\text {a }}$ Edição do guia PMBOK® de 2017 estabelece que os processos da área Gerenciamento do Cronograma são: definir das atividades, sequenciar as atividades, estimar os recursos das atividades, estimar as durações, desenvolver o cronograma e controlar o cronograma (PMI, 2017). A extensão para a construção do guia PMBOK® adiciona particularidades a essa área de ggerenciamento, como utilizar a técnica linha de balanço para melhor sequenciar as atividades, gerenciar as restrições inerentes aos projetos de construção. Como restrições, o guia cita datas e marcos, restrições de requerimentos legais, restrições de clima (devido exposição às intempéries), e restrições de inspeções, aprovações e permissões (PMI, 2016).

As principais funções do PMP classificam-se, de acordo com Coelho (2003), em básicas e secundárias. A primeira função básica do PMP é proteger a produção contra a incerteza, realizando o detalhamento das tarefas do nível dos processos ao nível das operações (BALLARD, 1997); (BALLARD; HOWELL, 1998); (BALLARD, 2000); (HAMZEH, 2009); analisando as restrições (BALLARD, 1997); (BALLARD; HOWELL, 1998); (BALLARD, 2000); (HAMZEH, 2009); e efetuando o desenho das operações através de estudos realizados antes da execução (BALLARD, 1997); (HAMZEH, 2009).

Coelho (2003) estabelece mais duas funções básicas, a integração entre níveis de planejamento, este último também proposto por Ballard e Howell (1998). Como funções secundárias, está a análise dos fluxos físicos; o gerenciamento de custos; e o planejamento e controle de segurança (COELHO, 2003).

\section{MÉTODO DA PESQUISA}

Foi realizada no presente trabalho uma pesquisa bibliográfica acerca do PMP e sua integração com a extensão para a construção do guia PMBOK® a fim de alcançar os objetivos do presente trabalho. Os critérios adotados foram analisar trabalhos específicos acerca do PMP e a extensão para a construção do guia PMBOK® de 2016.

\section{RESULTADOS}

Apesar do guia PMBOK® incorporar as melhores práticas para o gerenciamento de projetos, a extensão para a construção não engloba as práticas do LPS®, os processos da área de conhecimento Gerenciamento do Cronograma do PMBOK® estão relacionados ao cronograma de longo prazo do LPS $®$. Há interface vaga entre planejamento e execução, o planejamento de curto prazo (que é crítico do ponto de vista da execução) é habitualmente negligenciado e ineficientemente executado (KOSKELA; HOWELL, 2001).

Algumas medidas adicionadas à extensão para a construção do guia PMBOK® estão relacionadas com ações realizadas no PMP de projetos de construção, mas apresentam 
pouco detalhamento. Por exemplo, a extensão para a construção do guia incorpora a gestão de restrições (PMI, 2016), mas não engloba muitas categorias e não especifica a importância de sua gestão, representada pelo planejamento das responsabilidades e dos prazos de antecipação para a remoção das restrições (BALLARD, 2000).

Apesar da extensão para a construção do guia PMBOK® não atribuir os níveis de planejamento hierárquico de longo, médio e curto prazo, a estrutura do guia nas áreas de conhecimento contribuem em aspectos para a proposição do modelo desse trabalho, uma vez que as doze áreas da extensão para a construção do guia PMBOK® são integradas para gerenciar o projeto e atender premissas básicas de tempo, custo e qualidade. No Quadro são apresentadas as interações das áreas de conhecimento da extensão para a construção do guia PMBOK® com o nível de PMP.

\section{Quadro 1 - Interação entre o guia PMBOK® para a construção e o PMP}

\begin{tabular}{|c|c|c|}
\hline $\begin{array}{c}\text { Área de } \\
\text { conhecimento } \\
\text { (PMI, 2016) }\end{array}$ & Interação com PMP & Descrição da interação \\
\hline $\begin{array}{l}\text { Gerenciamento } \\
\text { da Integração } \\
\text { do Projeto }\end{array}$ & $\begin{array}{l}\text { Realizar o controle integrado de } \\
\text { mudanças }\end{array}$ & $\begin{array}{c}\text { Mudanças impactam todas as áreas do projeto, } \\
\text { principalmente prazo e custo, no PMP deve ser } \\
\text { operacionalizado essa integração. }\end{array}$ \\
\hline $\begin{array}{l}\text { Gerenciamento } \\
\text { do Escopo do } \\
\text { Projeto }\end{array}$ & $\begin{array}{l}\text { Solicitação de Mudanças de } \\
\text { escopo do Projeto }\end{array}$ & $\begin{array}{l}\text { No processo de monitoramento e controle do } \\
\text { escopo, é realizada a análise da necessidade de } \\
\text { mudanças no projeto, que devem ser } \\
\text { formalizadas e consideradas no PMP. }\end{array}$ \\
\hline \multirow{5}{*}{$\begin{array}{l}\text { Gerenciamento } \\
\text { do Cronograma } \\
\text { do Projeto }\end{array}$} & $\begin{array}{l}\text { Utilizar linha de balanço para o } \\
\text { planejamento do projeto }\end{array}$ & $\begin{array}{l}\text { Utilizar a linha de balanço agrega transparência } \\
\text { na integração entre o longo e médio prazo } \\
\text { (DAVE; SEPPÄNEN; MODRICH, 2016). }\end{array}$ \\
\hline & Gerenciar as restrições & $\begin{array}{c}\text { No PMP ocorre a análise de restrição } \\
\text { (BALLARD, 2000), e o guia cita restrições } \\
\text { inerentes aos projetos de construção: datas e } \\
\text { marcos, restrições de requerimentos legais, } \\
\text { restrições de clima e restrições de inspeções, } \\
\text { aprovações e permissões. }\end{array}$ \\
\hline & Utilizar métricas de controle & $\begin{array}{c}\text { O guia não cita métricas de PMP, cita } \\
\text { vagamente a análise de durações reais e } \\
\text { previstas, e produtividade real e prevista. O } \\
\text { indicador PPC cumpre essa função no LPS®, } \\
\text { que deve ser avaliado no PMP para incorporar } \\
\text { aprendizagem (COELHO, 2003; BALLARD; } \\
\text { HOWELL, 1998) }\end{array}$ \\
\hline & $\begin{array}{l}\text { Analisar riscos relacionados a } \\
\text { prazo }\end{array}$ & $\begin{array}{l}\text { Analisar riscos que impactam o prazo do } \\
\text { projeto e planejar respostas aos riscos no PMP } \\
\text { (WEHBE; HAMZEH, 2013). }\end{array}$ \\
\hline & $\begin{array}{c}\text { Gerenciar a curva de progresso } \\
\text { do prazo }\end{array}$ & $\begin{array}{l}\text { Analisar projeção da curva de progresso de } \\
\text { prazo em relação à linha de base de prazo (em } \\
\text { linha de balanço), e posteriormente, analisar } \\
\text { impacto da projeção e efetuar medidas de } \\
\text { revisão de desempenho, realizadas no PMP } \\
\text { (DAVE; SEPPÄNEN; MODRICH, 2016). }\end{array}$ \\
\hline
\end{tabular}

Fonte: Os autores 
SIBRAGEC - ELAGEC 2019 - de 23 a 25 de Outubro - LONDRINA - PR

Quadro 1 - Interação entre o guia PMBOK® para a construção e o PMP (cont.)

\begin{tabular}{|c|c|c|}
\hline $\begin{array}{l}\text { Área de } \\
\text { conhecimento } \\
\text { (PMI, 2016) }\end{array}$ & Interação com PMP & Descrição da interação \\
\hline \multirow[b]{2}{*}{$\begin{array}{l}\text { Gerenciamento do } \\
\text { Custo do Projeto }\end{array}$} & Orçamento planejado do projeto & $\begin{array}{c}\text { Fornece os custos estimados e orçados } \\
\text { para detalhamento dos pacotes de trabalho } \\
\text { no PMP. }\end{array}$ \\
\hline & $\begin{array}{l}\text { Controle do custo por relatório } \\
\text { de progresso e performance } \\
\text { (RPP) }\end{array}$ & $\begin{array}{l}\text { O controle do custo informa desvios de } \\
\text { custos e impacta em necessidades de } \\
\text { ações durante o PMP, pois uma das } \\
\text { funções é gerenciar custo (COELHO, } \\
\text { 2003). }\end{array}$ \\
\hline $\begin{array}{l}\text { Gerenciamento dos } \\
\text { Recursos do Projeto }\end{array}$ & $\begin{array}{l}\text { Planejamento dos recursos e } \\
\text { layout dos estoques; Gerenciar } \\
\text { as quantidades, validades dos } \\
\text { estoques, e manutenções } \\
\text { preventivas e corretivas de } \\
\text { equipamentos; Controlar a } \\
\text { produtividade e consumo dos } \\
\text { estoques. }\end{array}$ & $\begin{array}{l}\text { Forte interação com função do PMP de } \\
\text { analisar os fluxos físicos realizadas no } \\
\text { PMP (ALVES, 2000). }\end{array}$ \\
\hline $\begin{array}{l}\text { Gerenciamento da } \\
\text { Qualidade do } \\
\text { Projeto }\end{array}$ & $\begin{array}{l}\text { Requisitos da qualidade } \\
\text { planejados }\end{array}$ & $\begin{array}{l}\text { Os requisitos da qualidade podem atribuir } \\
\text { restrições que devem ser considerados no } \\
\text { PMP (FIREMAN, 2012). }\end{array}$ \\
\hline $\begin{array}{l}\text { Gerenciamento da } \\
\text { Qualidade do } \\
\text { Projeto }\end{array}$ & $\begin{array}{c}\text { Resultados do controle da } \\
\text { qualidade }\end{array}$ & $\begin{array}{l}\text { Durante a execução das atividades, há a } \\
\text { garantia da qualidade e realizado o } \\
\text { controle. Os resultados do controle da } \\
\text { qualidade devem ser analisados no PMP a } \\
\text { fim de evitar reincidência de erros na } \\
\text { produção e retrabalhos (FIREMAN, } \\
\text { 2012). }\end{array}$ \\
\hline $\begin{array}{l}\text { Gerenciamento da } \\
\text { Comunicação do } \\
\text { Projeto }\end{array}$ & Planejar comunicação & $\begin{array}{l}\text { Ter padronizado comunicação do projeto é } \\
\text { importante para o PMP, pois necessita de } \\
\text { reuniões periódicas com todos os } \\
\text { envolvidos e definir distribuição das } \\
\text { informações (COELHO, 2003). }\end{array}$ \\
\hline $\begin{array}{l}\text { Gerenciamento da } \\
\text { Comunicação do } \\
\text { Projeto }\end{array}$ & $\begin{array}{l}\text { Comunicação do controle do } \\
\text { desempenho do projeto }\end{array}$ & $\begin{array}{l}\text { É no PMP que é analisado projeção do } \\
\text { prazo, informado aos envolvidos e } \\
\text { planejado medidas de revisão de } \\
\text { desempenho (COELHO, 2003). }\end{array}$ \\
\hline $\begin{array}{l}\text { Gerenciamento de } \\
\text { Risco do Projeto }\end{array}$ & $\begin{array}{l}\text { Identificar, analisar, planejar as } \\
\text { respostas aos riscos e controlar }\end{array}$ & $\begin{array}{c}\text { Durante o PMP deve-se identificar, } \\
\text { analisar e planejar as respostas aos riscos; } \\
\text { e controlar os riscos identificados a médio } \\
\text { e longo prazo (WEHBE; HAMZEH, } \\
\text { 2013). O guia contribui com lista } \\
\text { informativa com as causas de riscos mais } \\
\text { comuns na construção. }\end{array}$ \\
\hline
\end{tabular}

Fonte: Os autores 
SIBRAGEC - ELAGEC 2019 - de 23 a 25 de Outubro - LONDRINA - PR

Quadro 1 - Interação entre o guia PMBOK® para a construção e o PMP (cont.)

\begin{tabular}{|c|c|c|}
\hline $\begin{array}{c}\text { Área de } \\
\text { conhecimento } \\
\text { (PMI, 2016) }\end{array}$ & $\begin{array}{c}\text { Interação com } \\
\text { PMP }\end{array}$ & Descrição da interação \\
\hline $\begin{array}{l}\text { Gerenciamento } \\
\text { das Aquisições } \\
\text { do Projeto }\end{array}$ & $\begin{array}{l}\text { Planejar a } \\
\text { análise fazer } \\
\text { ou comprar }\end{array}$ & $\begin{array}{l}\text { É realizada a análise de fazer com próprios recursos ou } \\
\text { subcontratar, ação realizada no longo prazo para recursos classe } \\
1 \text { (prazo de aquisição longo) e a médio prazo para recursos } \\
\text { classe } 2 \text { (prazo de aquisição menor do que } 30 \text { dias) e } 3 \text { (prazo de } \\
\text { aquisição curtos) (BERNARDES, 2001). }\end{array}$ \\
\hline $\begin{array}{l}\text { Gerenciamento } \\
\text { das Aquisições } \\
\text { do Projeto }\end{array}$ & $\begin{array}{l}\text { Administrar as } \\
\text { aquisições }\end{array}$ & $\begin{array}{l}\text { É no PMP que é realizado o gerenciamento da cadeia de } \\
\text { suprimentos e aquisições, ou seja, o acompanhamento do } \\
\text { processo de aquisição dos recursos e efetuadas ações em caso de } \\
\text { restrições no processo (BALLARD, 2000). }\end{array}$ \\
\hline $\begin{array}{l}\text { Gerenciamento } \\
\text { das Partes } \\
\text { Interessadas do } \\
\quad \text { Projeto }\end{array}$ & $\begin{array}{l}\text { Identificar, } \\
\text { planejar, } \\
\text { gerenciar e } \\
\text { controlar as } \\
\quad \text { partes } \\
\text { interessadas }\end{array}$ & $\begin{array}{l}\text { Partes interessadas podem promover restrições para o projeto e } \\
\text { no PMP devem ser monitoradas, representa uma nova categoria } \\
\text { de restrição a ser gerenciada. }\end{array}$ \\
\hline \multirow[t]{2}{*}{$\begin{array}{l}\text { Gerenciamento } \\
\text { da Saúde, } \\
\text { Segurança, } \\
\text { Proteção e meio } \\
\text { ambiente do } \\
\text { projeto }\end{array}$} & $\begin{array}{l}\text { Gerenciar } \\
\text { restrições de } \\
\text { saúde, } \\
\text { segurança, } \\
\text { proteção da } \\
\text { obra e meio } \\
\text { ambiente }\end{array}$ & $\begin{array}{l}\text { Essa área cita restrições importantes a serem gerenciadas no } \\
\text { PMP: 1) Relacionadas as características e restrições ambientais } \\
\text { e de segurança do local da obra (residentes adjacentes, } \\
\text { dificuldade de acesso, restrições de som); } \\
\text { 2) Restrições de construção de indústrias que especifica ensaios } \\
\text { e simulações de segurança; 3) As condições da execução do } \\
\text { projeto, como trabalho ao ar livre em climas extremos, } \\
\text { trabalhando com máquinas barulhentas, remoção de vegetação, } \\
\text { trabalhando em uma região com recursos culturalmente } \\
\text { significativos, trabalho em altura. 4) Requisitos de zoneamento e } \\
\text { sinalização de segurança e meio ambiente na análise dos fluxos } \\
\text { físicos. }\end{array}$ \\
\hline & $\begin{array}{l}\text { Realizar o } \\
\text { plano de } \\
\text { gerenciamento } \\
\text { de tráfego }\end{array}$ & $\begin{array}{l}\text { Define os controles a serem exercidos sobre o tráfego nas } \\
\text { proximidades do local do projeto, arranjos de entrada e saída } \\
\text { (incluindo verificações de segurança), acesso e saída para } \\
\text { veículos de emergência, etc. que tem relação com a função de } \\
\text { analisar os fluxos físicos do PMP (COELHO, 2003). }\end{array}$ \\
\hline $\begin{array}{l}\text { Gerenciamento } \\
\text { Financeiro do } \\
\text { Projeto }\end{array}$ & $\begin{array}{l}\text { Controlar o } \\
\text { fluxo de caixa } \\
\text { do projeto }\end{array}$ & $\begin{array}{l}\text { Uma das funções do PMP é gerenciar os custos, gerenciando o } \\
\text { cronograma em concordância com o monitoramento do fluxo de } \\
\text { caixa do financiamento da construção (COELHO, 2003). }\end{array}$ \\
\hline
\end{tabular}

Fonte: Os autores

\section{CONSIDERAÇÕES FINAIS}

Foram apontadas as interações e contribuições das doze áreas de conhecimento da extensão do guia PMBOK® na construção com o PMP. Notou-se que o PMP interage com as doze áreas de gestão de projetos, incorporando ações presentes em algumas áreas de conhecimento ou recebendo dados de entradas de outras. Isso aponta a importância gerencial do PMP para que um projeto.

A extensão do guia PMBOK® na construção prevê outras restrições não elencadas por autores primários acerca do PMP, como gerenciar restrições ambientais e de saúde no trabalho, gerenciar restrições relacionadas a requisitos de qualidade e de partes interessadas que possam comprometer o andamento da construção. 
Outra contribuição é ressaltar a gestão do controle integrado de mudanças pertencente a área de conhecimento Integração, fator que pode comprometer o prazo e custo do empreendimento e que pode ser gerenciado no PMP.

Ademais, além da gestão de restrições, o guia realça a importância da gestão de riscos no projeto, que pode comprometer o andamento da construção. $\mathrm{O}$ guia contribui com apêndice em seu livro composto por lista informativa com as causas de riscos mais comuns na construção, servindo como um banco de dados de possíveis riscos que podem ser utilizados no PMP.

\section{REFERÊNCIAS}

ALVES, T. da C. L. Diretrizes para a gestão dos fluxos físicos em canteiros de obras: proposta baseada em estudos de caso. 2000. 264f. Dissertação (Mestrado - Programa de PósGraduação em Engenharia Civil). Universidade Federal do Rio Grande do Sul, 2000.

BALLARD, G.; HOWELL, G. Shielding Production: Essential Step in Production Control. Journal of Construction Engineering and Management, v. 124, n. 1, p. 11-17, 1998. Disponível em: <https://bit.ly/2rQZF6P>.

BALLARD, G.; TOMMELEIN, I. Current Process Benchmark for the Last Planner System. Lean Construction Journal, p. 57-89, 2016. Disponível em: 〈https://bit.ly/2IukWKA〉.

BALLARD, H. G. Lookahead Planning: The Missing Link in Production Control. In: 5th Annual Conference of the International Group for Lean Construction, Anais...1997. Disponível em: <https://bit.ly/2rO23vO>.

BALLARD, H. G. The last planner system of production control. 2000. 192f. Doctor of Philosophy, University of Birmingham, Birmingham, 2000. Disponível em:

$<$ http://etheses.bham.ac.uk/4789/>.

BERNARDES, M. M. e S. Desenvolvimento de um modelo de planejamento e controle da produção para micro e pequenas empresas de construção. 2001. 294f. Dissertação (Mestrado - Programa de Pós-Graduação em Engenharia Civil). Universidade Federal do Rio Grande do Sul, 2001.

COELHO, H. O. Diretrizes e requisitos para o planejamento e controle da produção em nível de médio prazo na construção civil. 2003. 135f. Dissertação (Mestrado em Engenharia Programa de Pós-Graduação em Engenharia Civil. Universidade Federal do Rio Grande do Sul, Porto Alegre, 2003. Disponível em: 〈https://bit.ly/2L72ZDA〉.

DAVE, B.; SEPPÄNEN, O.; MODRICH, R.-U. Modeling Information Flows Between Last Planner and Location Based Management System. In: 24th Annual Conference of the International Group for Lean Construction, (IGLC-24), Boston, USA. Anais... Boston, USA: 2016. Disponível em: <http://iglc.net/Papers/Details/1321>.

FIREMAN, M. C. T. Proposta de método de controle integrado entre produção e qualidade com mensuração de perdas por making-do e pacotes informais. 2012. 179f. Dissertação (Mestrado - Programa de Pós-Graduação em Engenharia Civil). Universidade Federal do Rio Grande do Sul, 2012.

HAMZEH, F. R. Improving construction workflow - The role of production planning and control. 2009. 271f. Doctor of Philosophy in Engineering - Civil and Environmental Engineering, University of California, Berkeley, 2009. Disponível em: <https://bit.ly/2KuFQtJ>.

KOSKELA, L.; HOWELL, G. Reforming Project Management : the Role of Planning, Execution and Controlling. Proceedings of 9th International Group for Lean Construction Conference, p. 185-198, 2001. 
SIBRAGEC - ELAGEC 2019 - de 23 a 25 de Outubro - LONDRINA - PR

PMI. Construction extension to the PMBOK Guide. Newtown Square, Pennsylvania, EUA: Project Management Institute, 2016.

PMI. Um guia de conhecimento em gerenciamento de projetos. Guia PMBOK $6^{\circ}$. ed. EUA: Project Management Institute, 2017.

WEHBE, F. A.; HAMZEH, F. R. Failure mode and effect analysis as a tool for risk management in construction planning. In: 21st Annual Conference of the International Group for Lean Construction 2011, (IGLC-21), Fortaleza, Brazil. Anais... Fortaleza, Brazil: 2013. Disponível em: 〈https://bit.ly/2LbDIYT>. 